\title{
Una chiamata "vocazionale" a coltivare i propri talenti
}

\author{
Riccardo Gualdo
}

PUBBLICATO: 18 GIUGNO 2021

\section{Quesito:}

Un lettore chiede se sia appropriato tradurre con educazione professionale l'inglese vocational education, e propende per una resa più letterale: educazione vocazionale.

\section{Una chiamata "vocazionale" a coltivare i propri talenti}

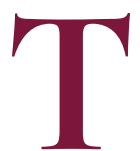

rovare un buon equivalente per i prestiti angloamericani non è facile. Le difficoltà aumentano con le parole inglesi di origine latina, che hanno un'aria di famiglia, ma non di rado una sfumatura di significato parzialmente o sensibilmente diversa dalle nostre. In questo secondo caso si è di fronte ai cosiddetti "falsi amici": commodity non significa comodità, bensì merce, morbid non è l'equivalente di morbido, ma di morboso, e cosi via. Quando il significato è solo in parte diverso, può succedere che l'accezione nuova del prestito vada ad aggiungersi a quelle già esistenti nella parola italiana: è stato cosi per realizzare, che ha acquisito il significato di 'rendersi conto' sul modello dell'inglese (to) realize.

A proposito della richiesta del nostro lettore, per prima cosa, va detto che il termine inglese vocational education è un tecnicismo della pedagogia; sarebbe quindi opportuno consultare qualche specialista prima di dare una risposta definitiva; e la chiusura - completa o parziale - di molte biblioteche rende più difficile consultare manuali ed enciclopedie del settore. Proverò dunque a rispondere limitandomi alle fonti che ho a disposizione: vocabolari, a stampa e in rete, e corpora testuali. Quest'ultima risorsa si rivela particolarmente utile con i termini rari - l'aggettivo vocazionale rientra senz'altro in questa categoria - e polirematici, cioè formati da più di un elemento lessicale.

La consultazione di un corpus di lingua italiana che raccoglie circa I2 miliardi di occorrenze, ricavate dalla rete (il TenTen corpus 2016. Italian, reperibile attraverso la raccolta di corpora SketchEngine), permette di osservare che le parole che compaiono con più frequenza associate all'aggettivo vocazionale sono tutte d'ambito religioso. Si va da pastorale - in assoluto la più frequente, con oltre 600 attestazioni, soprattutto nella combinazione pastorale vocazionale, in cui vocazionale è attributo di pastorale, sostantivo ('insieme dei mezzi per attuare il messaggio della Chiesa cristiana') - ad apostolato, passando per diocesano, eucaristico, missionario, parrocchiale, salesiano, seminario. Anche altre parole, che pure hanno nella lingua comune significati non direttamente vicini all'idea della "chiamata" da parte di Dio (ricordo che vocazione deriva dal lat. vocare 'chiamare') acquistano, in combinazione con vocazionale, una palpabile tramatura religiosa: è cosi per cammino e per giovanile (si noti che in quest'ultimo caso vocazionale è sostantivato: la vocazionale giovanile, con possibile ellissi di pastorale) cosi come per discernimento, che evoca immediatamente un percorso di riflessione (vedi, appunto, il "cammino") che conduca alla scelta del sacerdozio; piuttosto frequente è anche la combinazione con crisi: crisi vocazionale è sinonimo di crisi delle vocazioni.

I risultati sono diversi se cerchiamo vocational in un corpus di lingua inglese comparabile per dimensioni e selezione dei testi con quello italiano appena esaminato (TenTen corpus 2015. English): i nomi associati con più frequenza all'aggettivo sono rehabilitation 'riabilitazione', training, qualification, education, school e guidance, che significano, rispettivamente, 'addestramento, allenamento', 
'qualificazione', 'formazione' (vedi oltre), 'scuola', 'orientamento'.

Mentre in italiano esiste la figura del promotore o accompagnatore vocazionale, un sacerdote o un laico che assiste il giovane nel suo percorso, aiutandolo a maturare una decisione consapevole, nella vocational rehabilitation inglese c'è un counselor, che si occupa di assistere una persona con disabilità, congenita o accidentale, nel percorso di riabilitazione, eventualmente - se si tratta di un minorenne con l'assistenza di altri educatori e dei familiari adulti: negli Stati Uniti il Vocational Rehabilitation Counselor è una figura professionale riconosciuta dal sistema educativo; in Gran Bretagna a counselor si preferiscono assessor o teacher. Un altro significato attribuito alla vocational education, nel sistema educativo statunitense (l'inglese britannico preferisce learning o qualification), rinvia alle tecniche usate nella scuola, soprattutto qualche decennio fa, per comprendere a quale carriera scolastica gli studenti fossero più "portati" e indirizzare precocemente ai college più prestigiosi chi dimostrasse particolari attitudini. Oggi queste forme di selezione sono guardate con più sospetto, ma dubito che siano state del tutto abbandonate. È rimasto invece nell'uso statunitense il termine vocational schools per indicare corsi successivi alle scuole secondarie a indirizzo professionale, o anche corsi professionalizzanti - in genere della durata di due anni - per chi non si iscrive a college o a università. Su questo modello - ed è evidentemente il significato cui si riferisce il lettore - la comunità europea usa già dagli anni Settanta la formula vocational education and training per indicare corsi professionalizzanti successivi alle scuole superiori.

In entrambi i casi la vocation riguarda dunque le abilità della persona, che vanno recuperate perché indebolitesi o perché perdute per motivi accidentali, oppure portate alla luce con tecniche maieutiche; quest'ultima vocazione è poi indirizzata in vario modo nel sistema scolastico e professionale, con un'attenzione più spiccata verso le abilità pratiche.

Loscillazione tra vocational education e vocational training, in assoluto le combinazioni piu frequenti nel corpus di lingua inglese, suggerisce che l'attenzione, piuttosto che su vocational / vocazionale, su cui tornerò nelle conclusioni, va rivolta a education e atraining. In inglese la parola education, pur coprendo in larga misura la stessa area semantica dell'italiano educazione, ha un'accezione leggermente più ristretta, circoscritta prevalentemente al sistema educativo, meglio ancora allistruzione e alla formazione scolastica. L'equivalente inglese di "una persona educata" è piuttosto "a well-behaved person" che non "an educated person", espressione che corrisponde a "una persona istruita" o "una persona che ha avuto un'ottima formazione, che ha fatto buone scuole".

Torno a vocazionale: l'unica attestazione dell'aggettivo vocazionale nel maggiore dizionario storico della nostra lingua, il GDLI, rinvia alla raccolta Parole nuove, curata nel ig63 da Bruno Migliorini, e riguarda - appunto - la scuola. Grazie all'aiuto dei colleghi della consulenza linguistica dell'Accademia posso riportare la voce, che appare già nell'appendice all'ultima edizione del Dizionario moderno di Alfredo Panzini pubblicata nel I950. Migliorini aveva colto l'uso incipiente dell'aggettivo in àmbito pedagogico; ecco la sua definizione:

Vocazionale di, della vocazione, che rispetta la vocazione («scuola vocazionale»; che permette la scelta della carriera quando il giovane è in grado di riconoscere la propria vocazione). Der., ohimè, vocazionalitá.

Ma quel significato è rimasto ai margini della nostra lingua: tra i vocabolari sincronici, tra i maggiori dizionari sincronici, il Treccani registra solo il valore religioso, con l'esempio tendenza v. al sacerdozio; il GRADIT è più prudente, ma anche più vago, e spiega: "relativo alla vocazione; che favorisce e rispetta la vocazione: scelta, scuola vocazionale". 
Provo a tirare le fila del discorso, che si è fatto fin troppo lungo.

I) Litaliano vocazionale ha un'area d'uso prevalente non del tutto sovrapponibile a quella dell'inglese vocational; 2) la resa più precisa di education non è educazione, bensì formazione, istruzione.

Dunque sarebbe meglio, nel caso che stiamo esaminando, non tradurre vocational con vocazionale. Può essere interessante notare che i rapporti si invertono nella coppia vocazione / vocation: in inglese, vocation è usato prevalentemente in àmbito religioso: nel corpus TenTen 2015. English la parola è infatti associata - in ordine di frequenza - con gli aggettivi priestly, monastic, missionary, god-given, Salesian, Jesuit, Dominican, e cosi via; con il verbo (to) discern e con il sostantivo discernment (ricordo il discernimento vocazionale che ho segnalato in apertura), e con promoter, esatto omologo del nostro promotore. Viceversa, sappiamo bene che in italiano vocazione ha uno spettro d'uso più largo di quello strettamente religioso: si può ben dire, evidentemente per traslato (ma un traslato ben consolidato nella lingua comune), che qualcuno ha "la vocazione per lo sport" oppure per la musica, per lo spettacolo, o anche - perché no? - per la truffa.

Concludendo, in effetti educazione vocazionale appare una scelta poco convincente, perché cumula due calchi semantici, torcendo verso la formazione scolastica un aggettivo che ha una spiccata coloritura religiosa; ma anche educazione professionale non mi sembra una traduzione felicissima, perché coglie soltanto una parte del significato, suggerendo che la professione sia un obiettivo da raggiungere, mentre la vocazione - in senso ampio, non religioso - è un talento da scoprire e far emergere, semmai nutrendolo e plasmandolo in modo che si irrobustisca. Dunque sarebbe preferibile distinguere le due accezioni: formazione o istruzione attitudinale, o anche orientamento attitudinale, per l'azione pedagogica volta a riconoscere una vocazione, eformazione professionale per quando ci si riferisce ai corsi statunitensi di istruzione post secondaria.

\section{Cita come:}

Riccardo Gualdo, Una chiamata "vocazionale" a coltivare i propri talenti , "Italiano digitale", XVII, 2021/2 (aprile-giugno)

DOI: $10.35948 / 2532-9006 / 2021.9558$

Copyright 2021 Accademia della Crusca

Pubblicato con licenza creative commons CC BY-NC-ND 